\title{
Rational Design of Heterogeneous Catalysts for Biomass Conversion - inputs from computational chemistry
}

\author{
Romain Réocreux ${ }^{\mathrm{a}}$, Carine Michel $^{\mathrm{a}, *}$ \\ ${ }^{a}$ Univ Lyon, Ens de Lyon, CNRS UMR 5182, Université Claude Bernard Lyon 1, Laboratoire de Chimie, F-69342, Lyon, France
}

\begin{abstract}
The ever-growing development of biomass-based chemicals calls for a better understanding of the specificities of the corresponding catalytic processes. In this quest, ab initio modeling is a corner-stone that has proven its ability to rationalize the observed trends and then to propose novel catalysts design. Focusing on supported metal catalysts, we show that computational studies started a decade ago with alcohols and small polyols transformation, focusing on activity but also selectivity. Little by little, their scope has been extended to a variety of cellulosic-based chemicals such as levulinic acid or furanic molecules. During the last two years, it has also started to embrace lignin-derived chemicals, such as anisole, guaiacol, etc. Parallel to this scope expansion, the available methodologies have also progressed, triggered by the intrinsic difficulties of modeling biomass valorisation. In particular, improving the inclusion of the water solvent has drawn several groups to propose novel approaches.
\end{abstract}

Keywords: DFT, oxygenates, lignin, metal, catalysis, solvent

\section{Introduction}

With the necessary shift from petroleum-based feedstock to a biomass-based feedstock, the chemical industry needs to revise rapidly and extensively its production scheme. In addition, those new processes have to be sustainable, making catalysis a corner-stone of this revolution. To speed up the development of novel heterogeneous catalysts, ab initio modeling is a necessity, that provides in-depth mechanistic studies but also in silico screening of catalysts.[1] In here, we aim at highlighting the recent contributions of $a b$ initio modeling in the field of catalytic biomass valorization, with a restriction to metal supported catalysts as an illustrative domain.[2] With a special emphasize on the last three years, this review is divided into four parts. In a first short part, we describe the typical set-up employed to model a metal supported catalyst using periodic Density Functional Theory (DFT). Then, the contribution of computational chemistry to the valorisation of biomass is divided into two parts, following the type of biomass as shown in Figure 1. Biomass feedstock is made mainly of cellulose and hemicellulose that yield to molecules such as polyols and furanics. It has focused most of the experimental research effort in the last decade and we review in a dedicated part the main development made by computational chemistry in the domain. A smaller but not least interesting part is lignin. This heterogeneous aromatic polymer is a natural promising source of aromatics

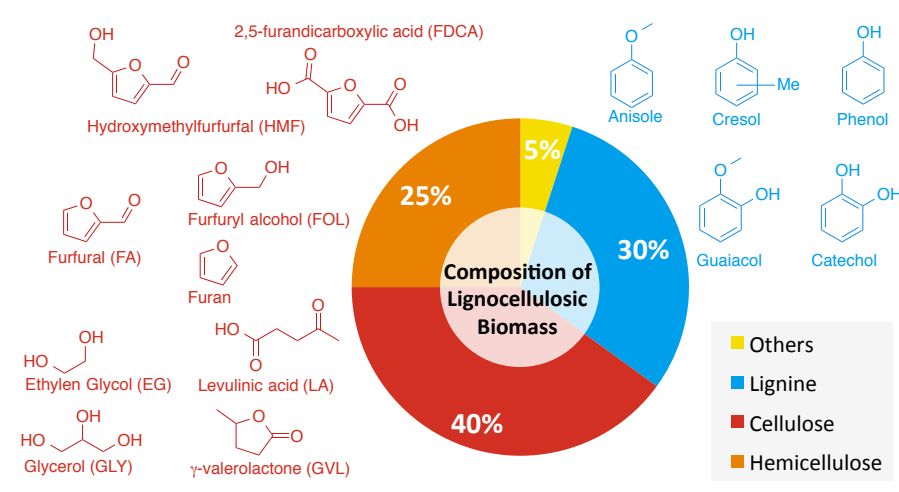

Figure 1: The biomass feedstock is mainly made of cellulose and hemicellulose but also of lignin at a smaller extent. The derived chemicals cited in this review are shown in red and in blue respectively.

but is highly challenging to convert into valuable chemicals. It attracts more and more interest as shown by the increasing number of studies dedicated to the catalytic processes to valorize lignin-based aromatic oxygenates. The corresponding contribution of DFT modeling is reviewed in a third part. Last, we discuss the main inputs provided by computational chemistry to the field of biomass valorization and draw some perspectives of progress in the domain.

\footnotetext{
* Corresponding author

Email address: carine.michel@ens-lyon.fr (Carine Michel)
} 


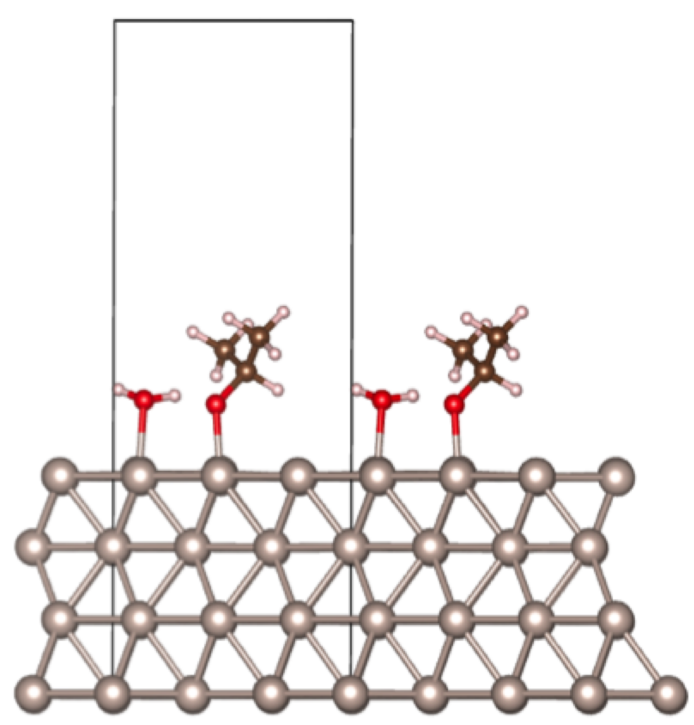

Figure 2: Example of a typical metallic slab model with a water and an isopropoxy intermediate adsorbed on the top layer. The cell is shown by a black rectangular and in repeated along the three directions of space during simulation.

\section{Fundamentals of $a b$ initio modeling applied to metal supported catalysts}

Combining Density Functional Theory (DFT) and periodic slab model of the most exposed facet (shown in Figure 2) is the work horse of computational studies of supported metal catalysts.[3] It provides an atomic "vision" of the reactions involved and even more importantly the energetics of the reaction paths including any secondary reactions. The knowledge of these paths allows predicting how the activity or the selectivity is affected by a change of metal for example.

Focused on the metallic active site, most of the studies neglect support effects, solvent effects and coverage effects on the overall catalytic process. Despite those limitations, they have proven their efficiency over the last decade in providing valuable knowledge to biomass valorization, and they are more and more advantageously coupled with micro-kinetic models. For a general review of theoretical simulations of heterogeneous catalysts, the reader can refer to one of the recent reviews.[3, 4]

\section{Polyols and other cellulosic-derived chemicals}

The first valuable inputs of computational chemistry were related to the conversion of cellulosic-derived chemicals such as polyols and furans. Those early studies were reviewed by two major groups. [5, 6]

To provide valuable knowledge, computational studies have to tackle huge reaction networks that involve potentially thousands of elementary steps. For instance, aqueous phase reforming of biomass consists in converting polyols into $\mathrm{CO}_{2}$ and $\mathrm{H}_{2}$. It requires to break C-C, C-H and

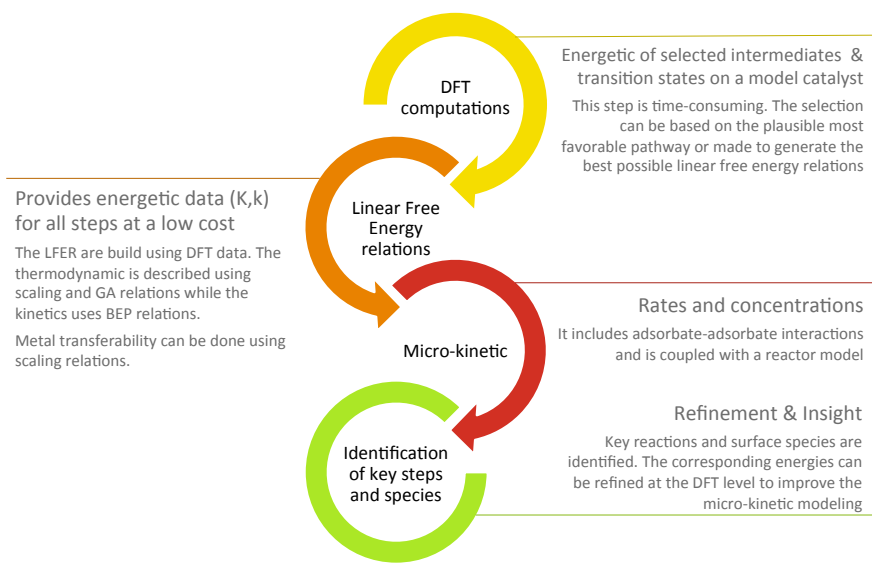

Figure 3: Workflow used to tackle extensive reactions network coupling ab initio modeling and micro-kinetics.

$\mathrm{O}-\mathrm{H}$ bonds of the starting material avoiding the C-O scission and includes also the water gas shift (WGS) reaction network. Providing a complete vision of the full reaction mechanism is highly challenging, even focusing on small polyols such as ethylene glycol and glycerol. The strategy that is usually implemented is illustrated in Figure 3. It consists in (i) extracting a representative subset of elementary steps (ii) computing at the DFT level the corresponding reaction energies and activation energies (iii) establishing linear free energy relations such as scaling relations [10] and group additivity (GA) relations[7] for the thermochemistry and Brønsted-Evans-Polaniy (BEP) relationships[8] for the kinetics (iv) implement a microkinetic model.

An optimal aqueous phase reforming (APR) catalyst has to show a good activity while being selective towards the C-C splitting. Several computational studies have focused on $\mathrm{Pt}(111)$, since, experimentally, $\mathrm{Pt}$ is the best known monometallic catalyst.[9] In line with what has been found on ethanol, $[10,11]$ the $\mathrm{C}-\mathrm{C}$ splitting in ethylene glycol (EG) and glycerol (GLY) is facilitated if CO is a product of the C-C scission.[12][13][14] In other words, a sufficient degree of dehydrogenation has to be reached to allow the $\mathrm{C}-\mathrm{C}$ scission. The $\mathrm{C}-\mathrm{O}$ bond scission is much less sensitive and always requires to overcome a high barrier (above $150 \mathrm{~kJ} / \mathrm{mol}$ ). However, the behavior of polyols cannot be systematically derived directly from that of the simple alcohols or alkanes. Indeed, the rate limiting step on $\mathrm{Pt}(111)$ is clearly the $\mathrm{C}-\mathrm{C}$ scission when considering ethanol,[11] while it is the first dehydrogenation step in polyols.[12][14] In addition, the $\mathrm{OH}$ scission is clearly affected by the presence of an intramolecular hydrogen bond on $\operatorname{Rh}(111)$ [15] and also on other late transition metals. [8] The identification of energetically feasible mechanisms can be automated as shown on glycerol.[16]

Computational chemistry can also deliver strong insight on the impact of the catalytic site. For instance, the role of less abundant sites can be assessed using other surfaces such as the (211) that is classically used to model 


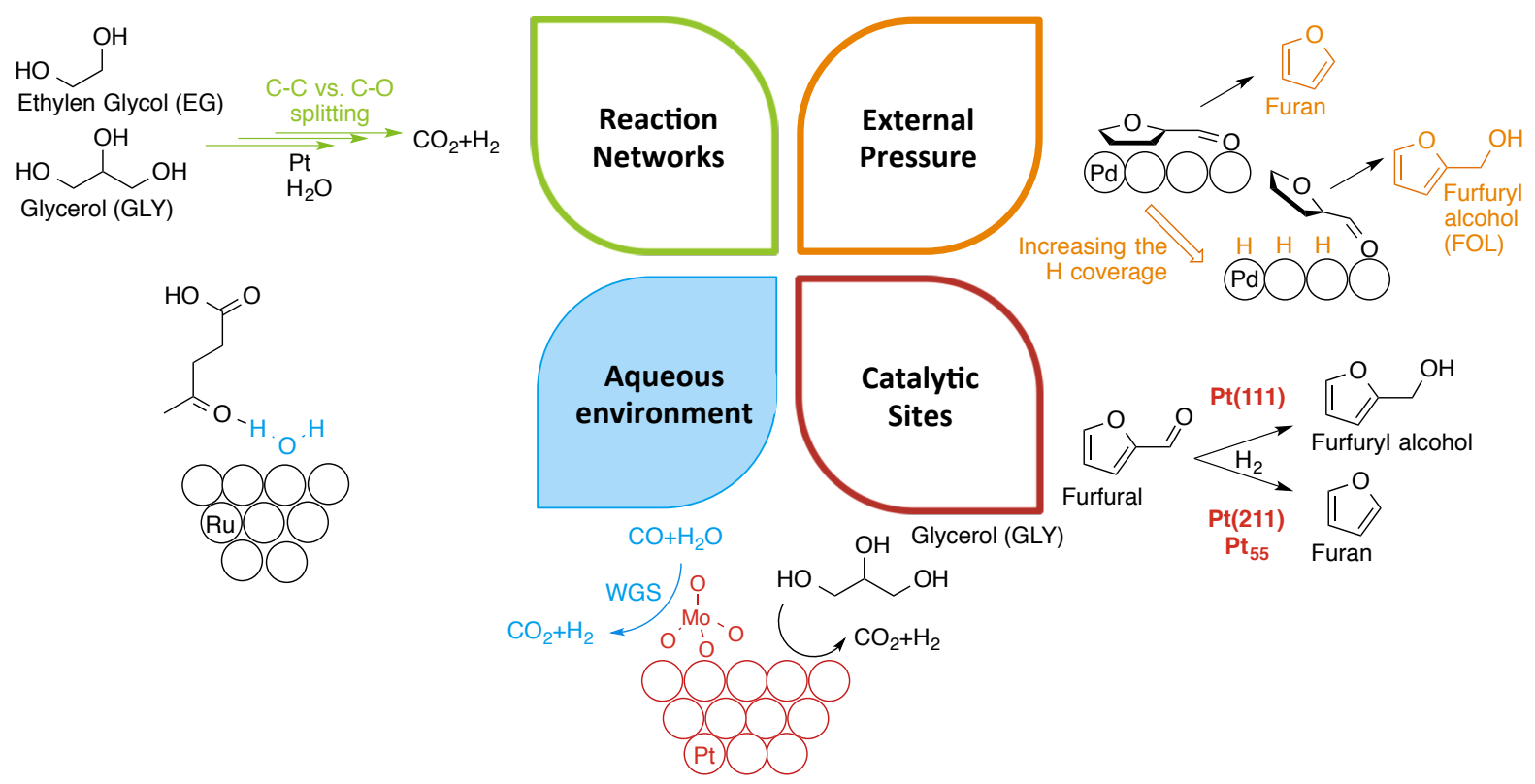

Figure 4: Computational chemistry can provided insights into several aspects: (i) extended reaction networks as shown on the APR of EG and GLY[12][13][14][16] (ii) nature of the catalytic sites with structure sensitivity[18] and bimetallics[20] (iii) effect of the water solvent on ketone hydrogenation catalyzed by $\mathrm{Ru}[21]$ (iv) effect of the external pressure of hydrogen on the selectivity.[26]

corner and edges. On Pt, Greeley and co-workers have found that those sites are more active towards ethylene glycol reforming but also more prone to be poisoned by $\mathrm{CO}$, an issue that may be mitigated by the WGS reaction in the aqueous phase as illustrated in Figure 4.[17] The influence of the active site on selectivity can also be addressed as nicely illustrated on the furanic transformation. On Pt, a combination of DFT computations and a microkinetic model have demonstrated for furfural (FAL) that the decarbonylation (yielding furan) prevails on small nanoparticles while the hydrogenation (yielding furfuryl alcohol, FOL) is favored on the large nanoparticles.[18] The C-C scission is strongly sensitive to the site structure and is favored on a $\mathrm{Pt}_{55}$ nanoparticle compared with a $\mathrm{Pt}(111)$ slab. Conversely, the oxidation of hydroxymethylfurfuran (HMF) into 2,5-furandicarboxylic acid (FDCA) is facilitated on nano-octahedron exposing mainly $\mathrm{Pd}(111)$ facets compared with nano-cubes that exhibit the more open (100) facet. This structure sensitivity has been rationalized by periodic DFT computations of the reaction mechanism: the key step is the alcohol dehydrogenation and it is eased on the (111) facet.[19]

Another strategy to tune catalytic sites is to use bimetallics. However rationalizing the underlying phenomena is particularly challenging. Computational chemistry is clearly a valuable tool in this context, as shown on glycerol reforming on Pt-Mo bimetallic.[20] The surface species Mo can easily form small molybdate clusters under the working conditions. This oxidized alloy ideally combines metallic Pt, which efficiently promotes the dehydrogenations steps and the C-C scission, and molybdenum oxide, which protects the catalyst from poisoning reducing the $\mathrm{CO}$ adsorption and favoring the WGS reaction (Figure 4).

Recently, most efforts have been made to better understand the influence of the water solvent. For instance, $\mathrm{Ru}$ supported catalysts are very efficient in converting levulinic acid into $\gamma$-lactone in water but not in tetrahydrofuran (THF) where no conversion is observed. This promoting effect of the water solvent is not observed in the case of $\mathrm{Pt}$ or Pd. The contrasted impact of the water molecules on the hydrogenation of the ketone function has been rationalized using periodic DFT computations together with a micro-solvation strategy,[21] which consists in adding one water molecules in the simulation box in this study (see Figure 2 and 4). The resulting hydrogen bond with the ketone completely modifies the hydrogenation pathways. It has no noticeable impact on the late metals such as $\mathrm{Pt}$ and $\mathrm{Pd}$ but it strongly modifies the stability of the alkoxy intermediate on the most oxophilic metal such as $\mathrm{Ru}$ or Co. On those oxophilic metals, the alkoxy intermediate is too stable in THF (or gas phase conditions), hampering the reaction. In water however it is destabilized by the co-adsorption with water, which prevents the catalyst from its poisoning. Moreover, the hydrogenation at the oxygen is facilitated by the presence of the co-adsorbed water. This study unravels why $\mathrm{Ru}$ is largely used in biomass valorization while it is barely used in gas phase.[22] To highlight this effect, the explicit inclusion of a chemisorbed water molecule was a necessity, as confirmed on a more recent study on methanol decomposition.[23]

Solvent effects can also lie in less specific interactions. 
Water has a strong dielectric constant that screens charges. This electrostatic effect can be efficiently taken into account by continuum models that are more and more developed and used in the field of computational catalysis. For instance, the systematic screening of a database of $\mathrm{C}_{x} \mathrm{O}_{y} \mathrm{H}_{z}$ fragments adsorbed on $\mathrm{Pt}(111)$ has shown recently that the inclusion of water as a continuum model mostly affect the orientation of the $\mathrm{C}-\mathrm{O}$ and $\mathrm{O}-\mathrm{H}$ bonds.[7] For example, in absence of a continuum water the $\mathrm{O}-\mathrm{H}$ bond in the $\mathrm{CHOH}$ fragment points down, toward the metallic surface, while it points up, once this continuum water solvent is included. This re-orientation is triggered by a better solvation of the $\mathrm{OH}$ group by up 10 to 20 $\mathrm{kJ} / \mathrm{mol}$. While the adsorption energies are barely affected by the inclusion of water, the $\mathrm{C}-\mathrm{H}$ bond scissions tend to be thermodynamically favored by the presence of water compared with the vacuum and the $\mathrm{O}-\mathrm{H}$ bond scission are mostly inhibited, due to the loss of the strong solvation of the $\mathrm{OH}$ group. Parallel to this work, the systematic study on the reaction mechanism of ethylene glycol (EG) reforming on $\mathrm{Pt}(111)$ has shown that the activation barrier of the $\mathrm{C}-\mathrm{H}$ scission is lowered by the inclusion of water as a continuum while that of the $\mathrm{OH}$ is not.[14]

More subtle effects such as that of $\mathrm{pH}$ can be also assessed. Recently, a computational study employing such a model has been able to rationalize the promoting effect of adding sodium formate to a formic acid solution to its decomposition into $\mathrm{H}_{2}$ and $\mathrm{CO}_{2} \cdot[24]$ This is a key reaction in several domains. In biomass, it can be used as a hydrogen transfer agent, limiting the utilization of $\mathrm{H}_{2}$. According to DFT computations that include the water solvent as a continuum model, the presence of chemisorbed sodium formate promotes the reaction and modifies the charge of the Pd surface. This promoting effect is nicely reproduced by a charged metallic slab together with a linear Poisson Boltzmann electrolyte model for the solvent and the counter charge. Thus, the role of the base could be also played by an appropriate support that could transfer charges to the Pd nanoparticles, a conclusion that opens the door to the rational design of new $\mathrm{Pd}$-based catalysts

Another important aspect is the role of the nature of the reacting gas such as $\mathrm{H}_{2}$. A first example is the switch in selectivity observed in glycerol conversion from 1,2-propanediol to lactic acid when changing the nature of the gas from $\mathrm{H}_{2}$ to an inert gas.[25] This effect was first rationalized by mechanistic investigations with the combination of DFT modeling and catalytic tests. Another example is the upgrade of furfural (FAL) under mild hydrogenation conditions using a pressure of $\mathrm{H}_{2}$. At the typical temperature of $180{ }^{\circ} \mathrm{C}$ and a pressure of $\mathrm{H}_{2}$ of 0.01 $1 \mathrm{~atm}$, the surface should be strongly covered by hydrogen $(0.7-0.9 \mathrm{ML})$. The presence of those co-adsorbates can modify the adsorption conformation of FAL and affect its overall conversion. This issue has been tackled by Wang et al.[26] FAL can adsorb in a flat conformation or in tilted conformations where the aldehyde function or the cycle points away from the surface. In the limit of low coverage, the flat conformation is the most stable since it maximizes the surface/molecule interactions. Once the hydrogen coverage is increased to $0.5 \mathrm{ML}$, it is destabilized to a greater extent than the tilted conformations (see Figure 4). This geometrical switch facilitates the hydrogenation of the carbonyl group compared with the decarbonylation. In this work, microkinetic models using the DFT-parametrized data have been set up. The low-coverage limit model predicts only decarbonylation to furan, whatever the conditions. The high coverage limit predicts a transition from decarbonylation to hydrogenation. This study demonstrates that the coverage in coadsorbed species may have to be taken into account to correctly predict the selectivity of a reaction.

\section{Aromatic Oxygenates and Lignin Valorisation}

With the recent development of new functionals designed to better account for vdW interactions (that represent about $50 \%$ of the total adsorption energy of aromatic compounds on metal surfaces), $[27,28]$ the $a b$ initio inspection of catalytic biomass conversion has opened to lignin derivatives for a couple of years now. The archetypical reaction studied in the field is the deoxygenation of guaiacol, an aromatic compound that exhibits two oxygenated moieties: an ether and a phenol group. In order to investigate the reactivity of each group independently, simpler models as anisole, phenol and cresols have also been considered in the literature (see Figure 1).

It is probably on the platinum catalyst that literature is most prolific in terms of detailed comparison between DFT and experimental data on the one hand, and the span of modeled conditions on the other hand (ultra high vacuum (UHV) to catalytic conditions). These studies have demonstrated that direct deoxygenations (namely direct $\mathrm{OH}$ and $\mathrm{OMe}$ removals) of aromatic oxygenates are impossible.[29-32] They indeed involve barriers of about $250 \mathrm{~kJ} / \mathrm{mol}$, higher than that of the reactant desorption itself (about $200 \mathrm{~kJ} / \mathrm{mol}$ ). The deoxygenation of the aromatic ring remains nevertheless energetically possible at the ether moiety. As shown in Figure 5, the dehydrogenation of the OMe group indeed yields $\mathrm{ArOCH}_{2}$ (Ar stands for aromatic ring) that decomposes into $\mathrm{ArO}+\mathrm{CH}_{x}+(1-$ $\mathrm{x}) \mathrm{H}$ (with $\mathrm{x}=0,1)$. The recombination of $\mathrm{ArO}$ and $\mathrm{CH}_{x}$ then yields $\mathrm{ArOCH}_{x}$ that can further get deformylated $(\mathrm{x}=1)$ or decarbonylated $(\mathrm{x}=0)$, illustrating the reducing character of carbonaceous species towards aromatic oxygenates [30, 31, 33] Conversely, the phenolic C-O bond is relatively recalcitrant under UHV conditions. The weakly activated dehydrogenation of $\mathrm{ArOH}$ to $\mathrm{ArO}$ even makes the situation worse since adsorbed phenoxy species (resp. catechoxy) show a cyclohexadienone (resp. benzoquinone) structure with a double, and thus strengthened, $\mathrm{C}=\mathrm{O}$ bond (see resonance structures for phenoxy in Figure 5).[31, 34, $58]$ In the case of guaiacol, similar reactions happen under UHV conditions to yield 1,2-benzoquinone with now two $\mathrm{C}=\mathrm{O}$ bonds. In this regard, the study by Hensley et al. 


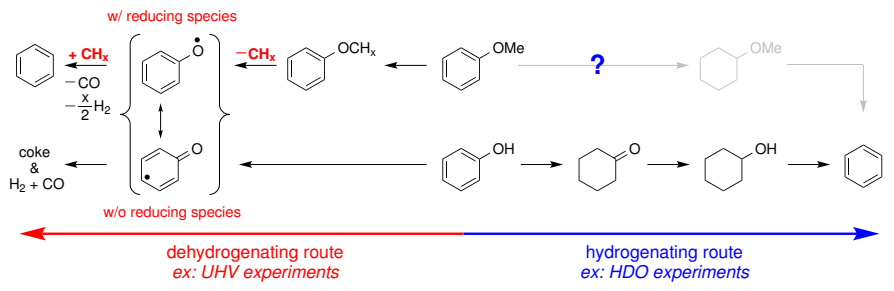

Figure 5: Orthogonal routes for aromatic ether and phenolic deoxygenation. The path in grey has not been evidenced computationally and is deduced from that of phenol.

combining DFT and state-of-the-art XPS measurements is particularly instructive. Unlike phenol or anisole, the vicinity of the two oxygenated groups significantly activates the aromatic $\mathrm{C}-\mathrm{C}$ bond on benzoquinone (activation barrier of only $40 \mathrm{~kJ} / \mathrm{mol}$ [58] vs $110-160 \mathrm{~kJ} / \mathrm{mol}$ for phenoxy [31]) leading to the full decomposition of the aromatic moiety on $\operatorname{Pt}(111)$. Under an atmosphere of hydrogen, the chemistry is however very different. The deoxygenation of anisole undoubtedly appears as being limited since the necessary preliminary dehydrogenation of the OMe group is then strongly limited by a pressure of hydrogen close to 1 bar. Under those conditions, the hydrogenolysis of the O-Me bond rather happens yielding phenol. In phenol, the $\mathrm{OH}$ removal becomes possible under a pressure of hydrogen. But first, the aromatic ring needs to be sufficiently hydrogenated to switch from a phenol to an alcohol reactivity. $[32,35]$ This can have serious consequences on the selectivity for aromatic compounds, even if it seems that a proper tuning of pressure, temperature and time of contact can limit the formation of saturated hydrocarbons.[32] To sum up, and according to the present comprehension based on computional modelling, the deoxygenation of lignin-like compounds on platinum follows orthogonal routes for ethers (dehydrogenation) and phenols (hydrogenolysis after partial hydrogenation of the cycle). It competes with the hydrogenation of the aromatic moiety, strongly favored by the platinum surface known as a good hydrogenation catalyst.

In the attempt to favor phenolic C-O bond cleavages, more oxophilic metals have been screened.[35-40] Lu et al. have for instance proved with a micro-kinetic analysis of wide reaction networks that guaiacol only yields catechol on $\mathrm{Pt}(111)$ whereas it leads to phenol on $\mathrm{Ru}(0001)$. [30, 38] Even with a pressure of hydrogen, ruthenium is indeed able to dehydrogenate OMe groups, a necessary step for ether deoxygenation, as aforementioned. Moreover it offers the possibility to directly break Ar-O bonds. This elementary step is admittedly rate determining but can be made easier considering step surfaces, for which the production of benzene is even predicted.[36, 37] Iron-based catalysts also have the ability to activate phenolic bonds, proving the close relationship between the metal oxophilicity and its propensity to activate C-O bonds.[35, 40] In spite of the resulting increased selectivity, conversions rarely compete with those found on platinum. An alternative therefore consists in diminishing the affinity of $\mathrm{Pt}$ for the aromatic ring. This can be achieved by alloying $\mathrm{Pt}$ with Mo and has proved to deoxygenate selectively benzaldehyde.[41] Other alloys or bimetallic systems have been investigated including $\mathrm{Pd} / \mathrm{Fe}$ with $\mathrm{Pd}$ dedicated to the homolysis of $\mathrm{H}_{2}$ and Fe to the deoxygenation of the aromatic compounds.[42] In spite of all, maximizing both the selectivity and conversion playing solely with the nature of the catalyst remains a difficult task. Fortunately, more complex catalytic systems involving co-catalysts and supports offer numerous other dimensions for the optimization of catalysts.

In particular, an avenue that is worth exploring is considering other reducing agents than adsorbed hydrogen $\left(\mathrm{H}_{2} \rightarrow 2 \mathrm{H}_{(a d s)}\right)$ to tackle the problem of chemoselectivity. The comparison between UHV experiments and DFT mechanistic investigations has demonstrated that carbonaceous species are reasonable reducing agents to deoxygenate aromatics without hydrogenating the aromatic cycle. $[31,33]$ In the context of the oxygenation of ligninderived aromatics, hydrogen would therefore appear as unnecessary. However, a lack of hydrogen has also proved to favor the dehydrogenation of the aromatic ring, leading to its total decomposition. It highlights the second and important role of hydrogen as an inhibitor of coke formation. The first role, namely the reducing one, can seemingly be replaced by other compounds to reduce the hydrogen content and avoid the hydrogenation of the aromatic ring. While carbonaceous species were playing that role under UHV conditions, they cannot be used under catalytic conditions since they would be readily hydrogenated to hydrocarbons that can easily desorb. Zn-decorated platinum surfaces offer a nice alternative.[43] They have proved experimentally to be able to selectively deoxygenate aromatic oxygenates. Such systems would be worth modeling in the future to get atomic insights on how to improve them. Eventually, supports can also provide a more chemoselective reducing character to the catalyst. Computational studies on $\mathrm{Ru} / \mathrm{TiO}_{2}$ and $\mathrm{Pt} / \mathrm{TiO}_{2}$ suggest that the adsorption of the aromatic ring happens on the metallic particle, while the direct deoxygenation is activated by defects on $\mathrm{TiO}_{2}$, which guest the oxygenated substituent.[44, 45]. Among those studies, the excellent work by Nelson et al. [44] combining experiments and theory deserves much attention. They have been able to rationalize, at the atomic level, the extraordinary activity and selectivity of $\mathrm{Ru} / \mathrm{TiO}_{2}$ when small metallic nanoparticles are used (a dozen of $\mathrm{Ru}$ atoms) in combination with water as a co-catalyst. Although the adsorption of water blocks the defect sites of $\mathrm{TiO}_{2}$ with surface hydroxyles, it opens the road to a novel mechanism involving the heterolysis of hydrogen into a proton on $\mathrm{TiO}_{2}$ and a hydride on the metal $\left(\mathrm{H}_{2} \rightarrow \mathrm{H}_{\left(T i O_{2}\right)}^{+}+\mathrm{H}_{(R u)}^{-}\right)$. This interfacial heterolysis therefore generates an acidic group on the support, involved in the activation of the oxygenated substituents as leaving groups, and a hydride that recombines with the deoxygenated aromatic ring. Small metal particles are believed 
to be the most efficient, since they expose only limited surface areas which are just large enough for the adsorption of the aromatic ring (only 4 surface atoms are needed for the adsorption of aromatic oxygenates on close packed surfaces [46]) but not sufficiently broad for the (de)hydrogenation of the ring to take place (albeit very likely on extended metal surfaces). This study questions the reliability of the model of extended pristine surfaces (both in computational chemistry and surface science) in the context of catalytic transformations of aromatic molecules, although they are genuinely attainable today in terms of computational power.[47]

\section{Achievement and perspectives of computational chemistry in biomass valorization}

Within this review, the abilities of computational chemistry at providing a better understanding have been underlined, distinguishing platform molecules derived from the (hemi)-cellulosic biomass and the ones derived from lignin. While ethylene glycol (EG) and anisole are very different in nature, the ability of a Pt catalyst to perform a deoxygenation obeys the same rationale. The formation of $\mathrm{CO}$ is an essential ingredient. In EG, the $\mathrm{C}-\mathrm{C}$ scission can occur easily once a sufficient dehydrogenation level is reached so that one of the dissociation product is CO.[12] Similarly, the Ar-OMe bond breaks once the Ar-OC intermediate is reached.[31] To favor the $\mathrm{C}-\mathrm{O}$ bond splitting over the $\mathrm{C}-\mathrm{C}$ one to preserve the number of carbon, the same strategy of catalyst modification can be used, adding Mo atoms to the Pt catalyst is a good strategy as illustrated on glycerol[20] as well as on aromatic oxygenates.[48]

Several lines of improvements are currently on going in computational chemistry applied to biomass valorization. A first objective in the field is to tackle large reactions networks. While the general workflow is well established (see Figure 3), some developments are still required to be able to go beyond glycerol and reach $\mathrm{C} 4$ to $\mathrm{C} 6$ sugars such as sorbitol or more complex lignin models. In addition, reactions that happen in the bulk liquid phase need to be included, together with their dependancies to $\mathrm{pH}$. The current development in kinetic modeling will help to reach soon such targets.[49] Another intense line of research is the improvement of the description of the environment surrounding the different adsorbates. It includes the effects of coverage, co-adsorption[50] or even water than can generate surface Brønsted acid sites or play a key role as a solvent. Continuum model approaches have been developed and implemented in several groups concomitantly recently.[51-53] In conjunction with DFT functionals corrected for dispersion such as $\mathrm{PBE}+\mathrm{dDsC}$, [28] they will become the new standard to computed energies. Since those continuum models do not include the contribution of specific interaction with hydrogen bonds, [54] a complementary approach to solvent effects is the explicit inclusion of few water molecules. However, this strategy as shown its limits in homogeneous phase where novel approaches are proposed.[55] Similar developments have to be done to describe reactions at the metal/water interface. Approaches based on molecular dynamics are currently proposed, with a necessary shift from ab initio molecular dynamics[56] towards a force field approach,[57] calling for better metallic surface/water force fields. Finally, a strong line of research shared with other areas in heterogeneous catalysis is the constant improvement of the geometrical and chemical description of the catalytic sites under working conditions. We have highlighted the role of the structure of the catalyst (the nature of the exposed facets), the impact of the hydrogen coverage on the reactivity of furfural or the adsorption of guaiacol,[50] the significance of additives, [43] the inclusion of the support,[44] etc. These improvements are only possible when tackled in conjunction with in situ and operando experimental characterizations, which are particularly challenging due to the use of high pressure and/or of the presence of liquid water as a solvent.

\section{Bibliography}

[1] J. K. Nørskov, T. Bligaard, J. Rossmeisl, C. H. Christensen, Towards the computational design of solid catalysts, Nature Chemistry 1 (2009) 37. doi: 10.1038/nchem.121.

[2] M. Besson, P. Gallezot, C. Pinel, Conversion of Biomass into Chemicals over Metal Catalysts, Chem. Rev. 114 (3) (2014) 1827-1870. doi:10.1021/cr4002269.

[3] K. Reuter, C. P. Plaisance, H. Oberhofer, M. Andersen, Perspective: On the active site model in computational catalyst screening, The Journal of Chemical Physics 146 (4) (2017) 040901. doi:10.1063/1.4974931.

[4] N. López, N. Almora-Barrios, G. Carchini, P. Błoński, L. Bellarosa, R. García-Muelas, G. Novell-Leruth, M. García-Mota, State-of-the-art and challenges in theoretical simulations of heterogeneous catalysis at the microscopic level, Catal. Sci. Technol. 2 (12) (2012) 2405. doi:10.1039/c2cy20384g.

[5] * J. W. Medlin, Understanding and controlling reactivity of unsaturated oxygenates and polyols on metal catalysts, ACS Catalysis 1 (10) (2011) 1284-1297. doi:10.1021/cs200336r.

[6] * N. Guo, S. Caratzoulas, D. J. Doren, S. I. Sandler, D. G. Vlachos, A perspective on the modeling of biomass processing, Energy \& Environmental Science 5 (5) (2012) 6703-6716. doi:10.1039/c2ee02663e.

[7] G. H. Gu, B. Schweitzer, C. Michel, S. N. Steinmann, P. Sautet, D. G. Vlachos, Group additivity for aqueous phase thermochemical properties of alcohols on pt(111), The Journal of Physical Chemistry C 121 (39) (2017) 21510-21519. doi: 10.1021/acs.jpcc. 7 b07340

[8] * J. Zaffran, C. Michel, F. Delbecq, P. Sautet, Towards more accurate prediction of activation energies for polyalcohol dehydrogenation on transition metal catalysts in water, Catalysis Science \& Technology 6 (17) (2016) 6615-6624, doi:10.1039/c6cy00865h.

[9] R. Davda, J. Shabaker, G. Huber, R. Cortright, J. Dumesic, A review of catalytic issues and process conditions for renewable hydrogen and alkanes by aqueous-phase reforming of oxygenated hydrocarbons over supported metal catalysts, Applied Catalysis B: Environmental 56 (1) (2005) 171 - 186, doi: 10.1016/j.apcatb.2004.04.027.

[10] P. Ferrin, D. Simonetti, S. Kandoi, E. Kunkes, J. A. Dumesic, J. K. Nø rskov, M. Mavrikakis, Modeling ethanol decomposition on transition metals: A combined application of scaling and brønsted-evans-polanyi relations, Journal of the American Chemical Society 131 (16) (2009) 5809-5815, pMID: 19334787. doi:10.1021/ja8099322. 
[11] * J. E. Sutton, D. G. Vlachos, Ethanol activation on closedpacked surfaces, Industrial \& Engineering Chemistry Research 54 (16) (2015) 4213-4225. doi:10.1021/ie5043374.

[12] M. Salciccioli, D. G. Vlachos, Kinetic modeling of Pt catalyzed and computation-driven catalyst discovery for ethylene glycol decomposition, ACS Catalysis 1 (10) (2011) 1246-1256. doi:10.1021/cs2003593.

[13] * B. Liu, J. Greeley, Decomposition pathways of glycerol via $\mathrm{CH}, \mathrm{OH}$, and $\mathrm{CC}$ bond scission on $\mathrm{Pt}(111)$ : A density functional theory study, The Journal of Physical Chemistry C 115 (40) (2011) 19702-19709. doi:10.1021/jp202923w.

[14] * M. Faheem, M. Saleheen, J. Lu, A. Heyden, Ethylene glycol reforming on $\mathrm{Pt}(111)$ : first-principles microkinetic modeling in vapor and aqueous phases, Catalysis Science \& Technology 6 (23) (2016) 8242-8256. doi:10.1039/c6cy02111e.

[15] J. Zaffran, C. Michel, F. Auneau, F. Delbecq, P. Sautet, Linear energy relations as predictive tools for polyalcohol catalytic reactivity, ACS Catalysis 4 (2) (2014) 464-468 doi:10.1021/cs4010503.

[16] S. Rangarajan, R. R. O. Brydon, A. Bhan, P. Daoutidis, Automated identification of energetically feasible mechanisms of complex reaction networks in heterogeneous catalysis: application to glycerol conversion on transition metals, Green Chem. 16 (2) (2014) 813-823. doi:10.1039/c3gc41386a.

[17] X.-K. Gu, B. Liu, J. Greeley, First-Principles Study of Structure Sensitivity of Ethylene Glycol Conversion on Platinum, ACS Catalysis 5 (4) (2015) 2623-2631. doi:10.1021/cs5019088.

[18] Q.-X. Cai, J.-G. Wang, Y.-G. Wang, D. Mei, Mechanistic insights into the structure-dependent selectivity of catalytic furfural conversion on platinum catalysts, AICHE Journal 61 (11) (2015) 3812-3824. doi:10.1002/aic.14902.

[19] * D. Lei, K. Yu, M.-R. Li, Y. Wang, Q. Wang, T. Liu, P. Liu, L.-L. Lou, G. Wang, S. Liu, Facet effect of single-crystalline $\mathrm{Pd}$ nanocrystals for aerobic oxidation of 5hydroxymethyl-2-furfural, ACS Catalysis 7 (1) (2017) 421-432. doi:10.1021/acscatal.6b02839.

[20] ** B. Liu, M. Zhou, M. K. Y. Chan, J. P. Greeley, Understanding polyol decomposition on bimetallic PtMo catalysts: a DFT study of glycerol, ACS Catalysis 5 (8) (2015) 4942-4950. doi:10.1021/acscatal.5b01127.

A complete study of the reforming of glycerol including scaling relations and BEP relations, alloy models, phase diagrams to establish the nature of the catalytic sites, etc.

[21] * * C. Michel, J. Zaffran, A. M. Ruppert, J. Matras-Michalska M. Jedrzejczyk, J. Grams, P. Sautet, Role of water on metal catalyst performance for ketone hydrogenation. a join experimental and theoretical study on levulinic acid conversion into gammavalerolactone, Chemical Communications 50 (83) (2014) 12450 12453, doi:10.1039/C4CC04401K.

A study showing the importance of including water as a microsolvant to properly assess the catalytic activity of transition metals

[22] C. Michel, P. Gallezot, Why is ruthenium an efficient catalyst for the aqueous-phase hydrogenation of biosourced carbonyl compounds?, ACS Catalysis 5 (7) (2015) 4130-4132, doi:10.1021/acscatal.5b00707.

[23] M. Garcia-Rates, R. Garcia-Muelas, N. Lopez, Solvation effects on methanol decomposition on $\operatorname{Pd}(111), \operatorname{Pt}(111)$, and Ru(0001), J. Phys. Chem. C 121 (25) (2017) 13803-13809. doi:10.1021/acs.jpcc.7b05545.

[24] ** P. Wang, S. N. Steinmann, G. Fu, C. Michel, P. Sautet, Key role of anionic doping for $\mathrm{H}_{2}$ production from formic acid on Pd (111), ACS Catalysis 7 (3) (2017) 1955-1959, doi:10.1021/acscatal.61303544.

The influence of the $\mathrm{pH}$ is rationalized on a reaction catalyzed by $\mathrm{Pd}$ in water: in presence of a base, the catalyst is partlally charged by the adsorption of the basic anion.

[25] F. Auneau, C. Michel, F. Delbecq, C. Pinel, P. Sautet, Unravelling the mechanism of glycerol hydrogenolysis over rhodium catalyst through combined experimental and theoretical investigations, Chem. Eur. J. 17 (50) (2011) 14288-14299. doi:10.1002/chem.201101318.

[26] ** S. Wang, V. Vorotnikov, D. G. Vlachos, Coverage-Induced Conformational Effects on Activity and Selectivity: Hydrogenation and Decarbonylation of Furfural on Pd(111), ACS CATALYSIS 5 (1) (2015) 104-112. doi:10.1021/cs5015145.

Combination of DFT calculations and micro-kinetics to rationalize the effect of the external pressure of hydrogen

[27] H. Yildirim, T. Greber, A. Kara, Trends in Adsorption Characteristics of Benzene on Transition Metal Surfaces: Role of Surface Chemistry and van der Waals Interactions, J. Phys. Chem. C 117 (2013) 20572-20583. doi:10.1021/jp404487z.

[28] S. Gautier, S. N. Steinmann, C. Michel, P. Fleurat-Lessard, $\mathrm{P}$. Sautet, Molecular adsorption at $\mathrm{Pt}(111)$. How accurate are DFT functionals?, Phys. Chem. Chem. Phys. 17 (2015) 2892128930. doi:10.1039/C5CP04534G

[29] K. Lee, G. H. Gu, C. A. Mullen, A. A. Boateng, D. G. Vlachos, Guaiacol Hydrodeoxygenation Mechanism on Pt(111): Insights from Density Functional Theory and Linear Free Energy Relations, ChemSusChem 8 (2015) 315-322. doi:10.1002/cssc.201402940.

[30] J. Lu, S. Behtash, O. Mamun, A. Heyden, Theoretical Investigation of the Reaction Mechanism of the Guaiacol Hydrogenation over a Pt(111) Catalyst, ACS Catal. 5 (2015) 2423-2435. doi:10.1021/cs5016244

[31] ** R. Réocreux, C. A. Ould Hamou, C. Michel, J. B. Giorgi, P. Sautet, Decomposition Mechanism of Anisole on Pt(111): Combining Single-Crystal Experiments and FirstPrinciples Calculations, ACS Catal. 6 (12) (2016) 8166-8178. doi:10.1021/acscatal.6b02253.

On $\mathrm{Pt}$, hydrogen is not needed to be able to perform selectively the deoxygenation of anisole to benzene. The chemoselective reducing agents are here atomic $\mathrm{C}$ or methylidene $\mathrm{CH}$.

[32] ** G. H. Gu, C. A. Mullen, A. A. Boateng, D. G. Vlachos, Mechanism of Dehydration of Phenols on Noble Metals via First-Principles Microkinetic Modeling, ACS Catal. 6 (2016) 3047-3055. doi:10.1021/acscatal.6b00776.

To deoxygenate aromatic oxygenates using hydrogen as a reducing agent, the aromatic ring first needs to be hydrogenated.

[33] C. A. Ould Hamou, R. Réocreux, P. Sautet, C. Michel, J. B. Giorgi, Adsorption and Decomposition of a Lignin $\beta$-O-4 Linkage Model, 2-Phenoxyethanol, on $\mathrm{Pt}(111)$ : Combination of Experiments and First-Principles Calculations, J. Phys. Chem. C 121 (18) (2017) 9889-9900. doi:10.1021/acs.jpcc.7b01099.

[34] H. Ihm, J. M. White, Stepwise Dissociation of Thermally Activated Phenol on Pt(111), J. Phys. Chem. B 104 (2000) 62026211. doi:10.1021/jp0005423.

[35] Q. Tan, G. Wang, A. Long, A. Dinse, C. Buda, J. Shabaker, D. E. Resasco, Mechanistic analysis of the role of metal oxophilicity in the hydrodeoxygenation of anisole, J. Catal. 347 (2017) 102-115. doi:10.1016/j.jcat.2017.01.008.

[36] C.-c. Chiu, A. Genest, A. Borgna, N. Rösch, Hydrodeoxygenation of Guaiacol over Ru(0001): A DFT Study, ACS Catal. 4 (2014) 4178-4188. doi:10.1021/cs500911j.

[37] * * C.-C. Chiu, A. Genest, A. Borgna, N. Rösch, C-O cleavage of aromatic oxygenates over ruthenium catalysts. A computational study of reactions at step sites, Phys. Chem. Chem. Phys. 17 (2015) 15324-15330. doi:10.1039/C5CP01027F.

Albeit difficult on extended flat $\mathrm{Ru}$ surfaces, the deoxygenation of phenoxy becomes possible on step surfaces

[38] J. Lu, A. Heyden, Theoretical investigation of the reaction mechanism of the hydrodeoxygenation of guaiacol over a $\mathrm{Ru}(0001)$ model surface, J. Catal. 321 (2015) 39-50. doi:10.1016/j.jcat.2014.11.003.

[39] Q. Tan, G. Wang, L. Nie, A. Dinse, C. Buda, J. Shabaker, D. E. Resasco, Different Product Distributions and Mechanistic Aspects of the Hydrodeoxygenation of m-Cresol over Platinum and Ruthenium Catalysts, ACS Catal. 5 (11) (2015) 6271-6283. doi:10.1021/acscatal.5b00765.

[40] A. J. R. Hensley, Y. Wang, J.-S. McEwen, Phenol Deoxygenation Mechanisms on $\mathrm{Fe}(110)$ and $\mathrm{Pd}(111)$, ACS Catal. 5 (2) (2015) 523-536. doi:10.1021/cs501403w. 
[41] A. M. Robinson, L. Mark, M. J. Rasmussen, J. E. Hensley, J. W. Medlin, Surface Chemistry of Aromatic Reactants on Pt- and Mo-Modified Pt Catalysts, J. Phys. Chem. C 120 (47) (2016) 26824-26833. doi:10.1021/acs.jpcc.6b08415.

[42] A. J. Hensley, Y. Wang, J.-S. McEwen, Adsorption of guaiacol on Fe (110) and Pd (111) from first principles, Surf. Sci. 648 (2016) 227-235. doi:10.1016/j.susc.2015.10.030.

[43] * D. Shi, L. Arroyo-Ramírez, J. M. Vohs, The use of bimetallics to control the selectivity for the upgrading of lignin-derived oxygenates: Reaction of anisole on Pt and PtZn catalysts, J. Catal. 340 (2016) 219-226. doi:10.1016/j.jcat.2016.05.020.

[44] ** R. C. Nelson, B. Baek, P. Ruiz, B. Goundie, A. Brooks, M. C. Wheeler, B. G. Frederick, L. C. Grabow, R. N. Austin, Experimental and Theoretical Insights into the Hydrogen-Efficient Direct Hydrodeoxygenation Mechanism of Phenol over Ru/TiO2, ACS Catal. 5 (11) (2015) 6509-6523. doi:10.1021/acscatal.5b01554.

Under the presence of water, the $\mathrm{Ru} / \mathrm{TiO}_{2}$ interface is able to perform the heterolysis of $\mathrm{H}_{2}$, which happens to be important for the activation of the oxygenated substituent first and then its substitution by an hydride.

[45] M. B. Griffin, G. A. Ferguson, D. A. Ruddy, M. J. Biddy, G. T. Beckham, J. A. Schaidle, Role of the Support and Reaction Conditions on the Vapor-Phase Deoxygenation of m-Cresol over $\mathrm{Pt} / \mathrm{C}$ and Pt/TiO2 Catalysts, ACS Catal. 6 (4) (2016) 27152727. doi:10.1021/acscatal.5b02868.

[46] R. Réocreux, M. Huynh, C. Michel, P. Sautet, Controlling the Adsorption of Aromatic Compounds on $\operatorname{Pt}(111)$ with Oxygenate Substituents: From DFT to Simple Molecular Descriptors, J. Phys. Chem. Lett. 7 (2016) 2074-2079. doi:10.1021/acs.jpclett.6b00612.

[47] J. Lu, M. Wang, X. Zhang, A. Heyden, F. Wang, $\beta-O-4$ Bond Cleavage Mechanism for Lignin Model Compounds over Pd Catalysts Identified by Combination of First-Principles Calculations and Experiments, ACS Catal. 6 (8) (2016) 5589-5598. doi:10.1021/acscatal.6b00502.

[48] A. M. Robinson, L. Mark, M. Rasmussen, J. E. Hensley, J. Medlin, Surface Chemistry of Aromatic Reactants on Pt and Mo-Modified Pt Catalysts, J. Phys. Chem. C in review. doi:10.1021/acs.jpcc.6b08415.

[49] C. F. Goldsmith, R. H. West, Automatic generation of microkinetic mechanisms for heterogeneous catalysis, The Journal of Physical Chemistry C 121 (18) (2017) 9970-9981. doi:10.1021/acs.jpcc.7b02133.

[50] * G. A. Ferguson, V. Vorotnikov, N. Wunder, J. Clark, K. Gruchalla, T. Bartholomew, D. J. Robichaud, G. T. Beckham, Ab Initio Surface Phase Diagrams for Coadsorption of Aromatics and Hydrogen on the Pt(111) Surface, J. Phys. Chem. C 120 (46) (2016) 26249-26258. doi:10.1021/acs.jpcc.6b07057.

[51] M. Faheem, S. Suthirakun, A. Heyden, New implicit solvation scheme for solid surfaces, The Journal of Physical Chemistry C 116 (42) (2012) 22458-22462. doi:10.1021/jp308212h.

[52] K. Mathew, R. Sundararaman, K. Letchworth-Weaver, T. A. Arias, R. G. Hennig, Implicit solvation model for densityfunctional study of nanocrystal surfaces and reaction pathways, The Journal of Chemical Physics 140 (8) (2014) 084106. doi: $10.1063 / 1.4865107$.

[53] M. Garcia-Ratés, N. López, Multigrid-based methodology for implicit solvation models in periodic dft, Journal of Chemical Theory and Computation 12 (3) (2016) 1331-1341, pMID: 26771105. doi:10.1021/acs.jctc.5b00949.

[54] S. K. Iyemperumal, N. A. Deskins, Evaluating solvent effects at the aqueous/Pt(111) interface, ChemPhysChem 18 (16) (2017) 2171-2190. doi:10.1002/cphc.201700162.

[55] S. H. Mushrif, V. Vasudevan, C. B. Krishnamurthy, B. Venkatesh, Multiscale molecular modeling can be an effective tool to aid the development of biomass conversion technology: A perspective, Chemical Engineering Science 121 (2015) 217-235. doi:10.1016/j.ces.2014.08.019.

[56] L. Bellarosa, R. García-Muelas, G. Revilla-López, N. López,
Diversity at the Water-Metal Interface: Metal, Water Thickness, and Confinement Effects, ACS Cent. Sci. 2 (2016) 109-116. doi:10.1021/acscentsci.5b00349.

[57] S. N. Steinmann, P. Sautet, C. Michel, Solvation free energies for periodic surfaces: comparison of implicit and explicit solvation models, Physical Chemistry Chemical Physics 18 (46) (2016) 31850-31861, doi:10.1039/c6cp04094b.

[58] ** A. J. R. Hensley, C. Wockel, C. Gleichweit, K. Gotterbarm, C. Papp, H.-P. Steinruck, Y. Wang, R. Denecke, J.S. McEwen, Identifying the thermal decomposition mechanism of guaiacol on $\mathrm{Pt}(111)$ : an integrated X-ray photoelectron spectroscopy and density functional theory study, The Journal of Physical Chemistry C, in press, doi:10.1021/acs.jpcc.7b10006

This study gives a detailed decomposition mechanism of guaiacol under UHV conditions. Using DFT and the most advanced synchrotron-based XPS measurements, this study provides key intermediates and significant insight on lignin-derived aromatic oxygenates. It also provides very nice perspectives in terms of future DFT/XPS comparisons for complex surface reactions 